\title{
Nanoscale surface domain formation on the $+z$ face of lithium niobate by pulsed ultraviolet laser illumination
}

\author{
C. E. Valdivia, ${ }^{\text {a) }}$ C. L. Sones, J. G. Scott, S. Mailis, and R. W. Eason \\ Optoelectronics Research Centre, University of Southampton, Southampton, SO17 1BJ, United Kingdom \\ D. A. Scrymgeour and V. Gopalan \\ Materials Research Laboratory, Pennsylvania State University, University Park, Pennsylvania 16802 \\ T. Jungk and E. Soergel \\ Institute of Physics, University of Bonn, Wegelerstrasse 8, 53115 Bonn, Germany \\ I. Clark \\ CCLRC Rutherford Appleton Laboratory, Chilton, Didcot, Oxfordshire, OX11 OQX, United Kingdom
}

(Received 10 September 2004; accepted 9 November 2004; published online 6 January 2005)

\begin{abstract}
Single-crystal congruent lithium niobate samples have been illuminated on the $+z$ crystal face by pulsed ultraviolet laser wavelengths below $(248 \mathrm{~nm})$ and around $(298-329 \mathrm{~nm})$ the absorption edge. Following exposure, etching with hydrofluoric acid reveals highly regular precise domain-like features of widths $\sim 150-300 \mathrm{~nm}$, exhibiting distinct three-fold symmetry. Examination of illuminated unetched areas by scanning force microscopy shows a corresponding contrast in piezoelectric response. These observations indicate the formation of nanoscale ferroelectric surface domains, whose depth has been measured via focused ion beam milling to be $\sim 2 \mu \mathrm{m}$. We envisage this direct optical poling technique as a viable route to precision domain-engineered structures for waveguide and other surface applications. (c) 2005 American Institute of Physics.
\end{abstract}

[DOI: $10.1063 / 1.1849414]$

Lithium niobate is a ferroelectric material ideally suited to a range of nonlinear, electro-optic, and piezoelectric interactions. Nonlinear interactions such as frequency conversion can be controlled and enhanced through the process of quasiphase-matching (QPM) which necessitates periodic domain inversion to reverse the sign of the second-order nonlinear susceptibility, $\chi^{(2)}$, after each coherence length. Methods employed for engineering the ferroelectric domain structure thus far include $\mathrm{Li}_{2} \mathrm{O}$ outdiffusion, ${ }^{1}$ proton-exchange, ${ }^{1}$ Ti-indiffusion, ${ }^{2}$ and electron beam irradiation. ${ }^{3}$ Electric-field poling, ${ }^{4}$ however, is currently the preferred and perhaps most reliable method of domain engineering, capable of fabricating bulk periodic structures down to the approximately few microns scale. ${ }^{5}$ Recently, high voltage atomic force microscopy has been used to demonstrate submicron features by scanning large, highly inhomogeneous electric fields across ferroelectric surfaces, ${ }^{6}$ but suffers from slow processing due to its serial nature. Other recent but still experimental techniques, such as coercive field reduction via illumination by visible $^{7}$ or $\mathrm{UV}^{8,9}$ light with concurrent electric-field poling, may well be able to produce submicron high quality domain structures, but these have yet to be reported.

Several current and future applications of these ferroelectric nonlinear materials such as efficient first-order QPM UV second harmonic generation, electro-optic Bragg gratings, or photonic crystal structures require submicron domain periods. However, these nanoscale individual domain widths fall outside the current limit of routine fabrication capability. Additionally, simple, single-step and inexpensive fabrication techniques are desirable if possible. In this letter, we demonstrate such a direct optical poling technique for congruent lithium niobate using light below $(248 \mathrm{~nm})$ and

\footnotetext{
${ }^{a)}$ Electronic mail: cev@orc.soton.ac.uk
}

near $(\sim 300 \mathrm{~nm})$ the UV absorption edge. The laser-modified domain manipulated materials have been investigated by both differential chemical etching and scanning force microscopy (SFM).

Optical poling is defined here as domain inversion by direct pulsed laser illumination, and we have chosen two wavelength regions to explore the technique in detail. At $248 \mathrm{~nm}$, the absorption depth is very small, certainly below $1 \mu \mathrm{m}$. Near $300 \mathrm{~nm}$ wavelengths, the transmission starts to increase, and we have therefore used light of wavelengths between 298 and $329 \mathrm{~nm}$ to investigate the effects of controlled exposure in near-surface regions. Laser illumination was via either a $\mathrm{KrF}$ excimer laser (Lambda Physik LPX $200)$ at $248 \mathrm{~nm}(\sim 20 \mathrm{~ns}$ pulses$)$, or a frequency-doubled $Q$-switched Nd:YAG laser (Continuum Powerlite 8000) pumping a dye laser (Sirah PrecisionScan) which was also frequency doubled, generating millijoule level pulses between 298 and $329 \mathrm{~nm}$ ( $\sim 7 \mathrm{~ns}$ pulses). For both laser systems, the beam passed through an aperture to select a small region of improved spatial uniformity and this area was subsequently imaged onto the $+z$ face of a 500- $\mu$ m-thick single crystal congruent lithium niobate sample (Crystal Technology, USA). We have separately investigated the effects of $248 \mathrm{~nm}$ exposure on the $-z$ face, and these results will be reported elsewhere. ${ }^{10}$

For incident energy densities near the single-pulse ablation threshold of lithium niobate (of order $0.5 \mathrm{~J} \mathrm{~cm}^{-2}$ ), uniform UV irradiation produces little surface damage. As energy densities increase however, multiple pulse exposures of 5 pulses of $298 \mathrm{~nm}$ light at an energy density per pulse of $\sim 10 \mathrm{~J} \mathrm{~cm}^{-2}$ leads to ablation depths of $\sim 1.5 \mu \mathrm{m}$. Interestingly, the wide range of fluences $\left(\sim 0.5-10 \mathrm{~J} \mathrm{~cm}^{-2}\right)$ tested at $298 \mathrm{~nm}$ produce similar results when examined by both selective etching and SFM (discussed below), providing a 


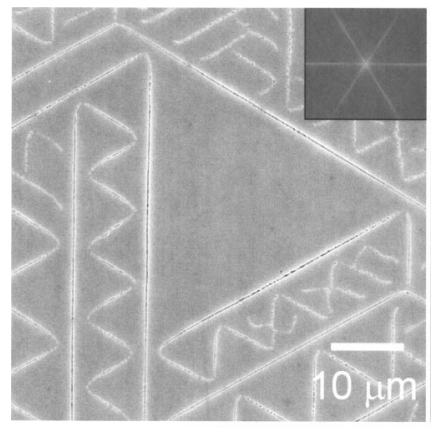

(a)

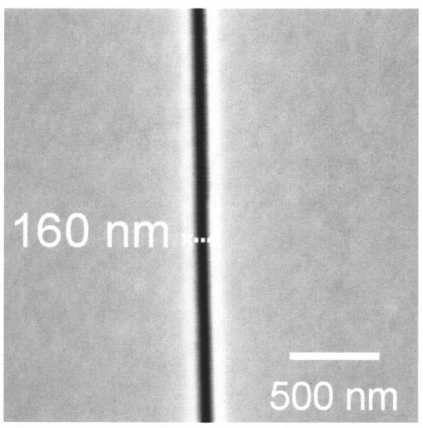

(b)
FIG. 1. SEM micrographs of the $+z$ face of lithium niobate exposed to $298 \mathrm{~nm}$ light ( $\sim 0.7 \mathrm{~J} \mathrm{~cm}^{-2}, 5$ pulses$)$ and subsequently etched in HF acid for $30 \mathrm{~min}$. (a) Etched trenches aligned parallel and at $\pm 60^{\circ}$ to the $y$ axis (vertical). The inset shows the 2D Fourier transform of the etched pattern, clearly demonstrating the three-fold symmetry. (b) Detail from (a), showing a single continuous etched line feature of good uniformity.

broad processing window for this direct optical poling effect.

Following exposure of the $+z$ crystal faces, the irradiated lithium niobate samples were placed in a hydrofluoric (HF) acid bath at room temperature for 30-60 min. Chemical etching using HF acid is well known to selectively etch the $-z$ crystalline face, with no effect on the $+z$ face. Scanning electron microscopy (SEM) images of the resulting structures on the $+z$ face show within the UV-irradiated regions a self-organized pattern of etched trenches [Fig. 1(a)] that run parallel and at $\pm 60^{\circ}$ angles to the crystallographic $y$ axis, indicating that their formation is dictated by the underlying $3 \mathrm{~m}$ crystal symmetry. The inset in Fig. 1(a) shows the twodimensional (2D) Fourier transform, confirming that growth occurs along these crystalline directions only.

The patterned features seen in Fig. 1(a) are very similar to those obtained via light induced frustrated etching (LIFE) experiments performed in Fe:doped samples of lithium niobate $^{11}$ using long exposures of the $-z$ crystal face to $\mathrm{cw}$ visible laser light in the presence of an HF etch environment. The three-fold symmetry, filling in of the available space by triangular zig-zag patterns and failure of any lines to cross as seen in the LIFE experiments is entirely consistent with the present case. This may well indicate a similar underlying physical mechanism, which in the cw experiments was thought to be a charge-mediated effect generated by the Fe dopant.

Analysis of all the SEM micrographs showed that the trenches illustrated by Fig. 1(b) had widths that fell within the range of $150-300 \mathrm{~nm}$, with most line features uniform along their lengths. The longest lines, extending up to hundreds of microns, were seemingly limited only by the spot size of the UV beam incident on the sample surface. Figure 1(b) shows a close-up of one of these pulsed UV laserinduced line features, and it is clear that the trench is small ( $\sim 160$-nm width), highly uniform, straight, and has been etched out of the surrounding $+z$ face, indicating the likely presence of $a-z$ oriented domain feature.

The depth of these trenches was evaluated by HF etching, followed by focused ion beam (FIB) milling. Because the etch rate of the $-z$ face of lithium niobate in $48 \% \mathrm{HF}$ solution is $\sim 0.8 \mu \mathrm{m} \mathrm{h}^{-1}$ at room temperature, ${ }^{12}$ etching was undertaken for $18 \mathrm{~h}$ to ensure that the maximum etch depth had been reached. Sideways etching also occurs over this length of time, producing broadening of the trench width so

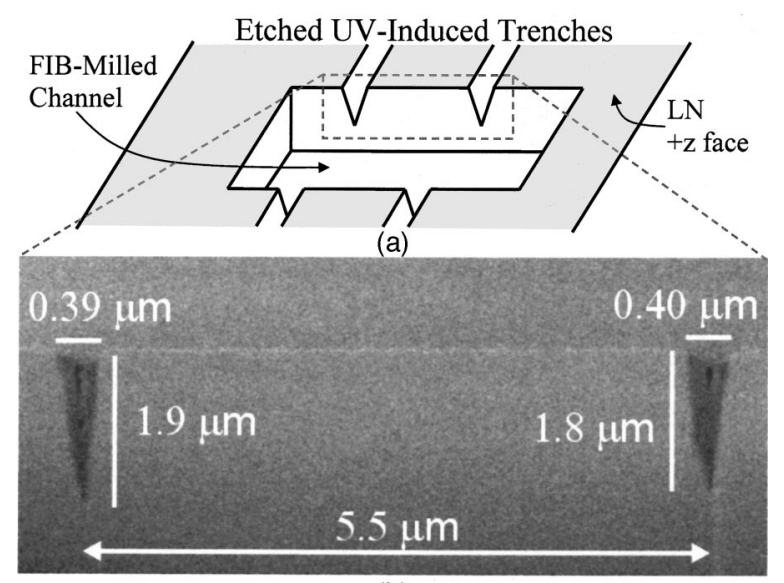

(b)

FIG. 2. (a) Diagram of a FIB-milled channel crossing two etched UVinduced trenches. (b) FIB micrograph of a fabricated area, corresponding to the region shown from (a), viewed at a $45^{\circ}$ angle. The etched trenches are shown to extend to $1.9 \mu \mathrm{m}$ depths; width broadening is also observable, as compared to the measured widths $(150-300 \mathrm{~nm})$ after shorter etch durations. The two trenches, formed by a large-area exposure of $298 \mathrm{~nm}$ light $\left(\sim 1.5 \mathrm{~J} \mathrm{~cm}^{-2}, 2\right.$ pulses), were revealed by selective chemical etching in $\mathrm{HF}$ acid for $18 \mathrm{~h}$.

that the FIB-measured widths of $\sim 400 \mathrm{~nm}$ do not necessarily reflect the minimum widths of these features.

Following etching, a channel was milled across the trenches, depicted in Fig. 2(a), using a FIB (FEI, Bristol, UK). The same FIB was used to image the milled channel at an angle of $45^{\circ}$, shown in Fig. 2(b). From this close-up view of the cross-sectional depth of the trench features, it is apparent that they extend to a depth approaching $2 \mu \mathrm{m}$. A comparison can be made here between these triangular shaped features and electric-field periodically poled surface domains seen via $y$-face etching studies, which extend to depths of order $10 \mu \mathrm{m} .^{13,14}$ The close similarity suggests that these etched trenches indicate that UV illumination has induced domain-inverted material $(-z$ orientation) within the background $+z$ face.

Irradiation of the $+z$ face was undertaken at many different wavelengths, including the fixed wavelength of $248 \mathrm{~nm}$, which corresponds to strong optical absorption in lithium niobate, and 298-329 nm, which spans the optical absorption band-edge. The features revealed by the selective chemical etching, however, were only observed for the wavelengths 248 and $298-306 \mathrm{~nm}$. $248 \mathrm{~nm}$ laser light is absorbed very near to the surface of the crystal, and is expected to have a very strong interaction with the material over a depth of possibly $1 \mu \mathrm{m}$ or less. For wavelengths shorter than $300 \mathrm{~nm}$, the transmission of light through a $500-\mu \mathrm{m}$-thick sample of lithium niobate is effectively zero, while the $50 \%$ transmission point occurs for a value of $328 \mathrm{~nm}$. For wavelengths longer than $306 \mathrm{~nm}$, it is possible that similar patterns would appear on the surface of the crystals for higher incident laser fluences, but these were not apparent up to the maximum fluences used of $7 \mathrm{~J} \mathrm{~cm}^{-2}$ per pulse in our studies.

At least a small amount of surface ablation by the incident laser light appeared to be a prerequisite for the formation of these self-organized features. In cases where no ablation appeared, HF acid treatment has not been observed to reveal the self-organized patterns. This may indicate a possible mechanism for the formation of these features, such as $\mathrm{Li}_{2} \mathrm{O}$ outdiffusion induced by the local surface heating from to AIP license or copyright; see http://apl.aip.org/apl/copyright.jsp 


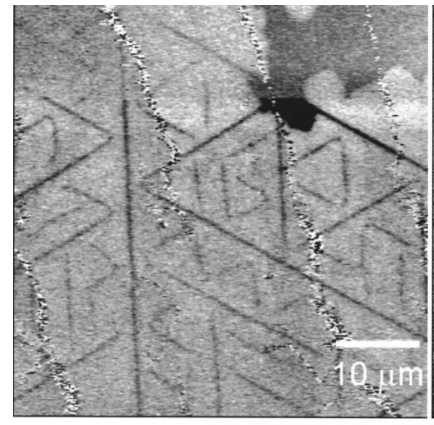

(a)

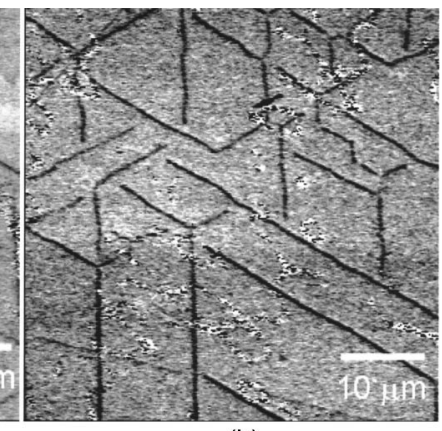

(b)
FIG. 3. SFM scans showing amplitude response following exposure of the $+z$ face to pulsed UV light at wavelengths of (a) $298 \mathrm{~nm}\left(3 \mathrm{~J} \mathrm{~cm}^{-2}, 5\right.$ pulses) and (b) $248 \mathrm{~nm}\left(0.3 \mathrm{~J} \mathrm{~cm}^{-2}, 20\right.$ pulses). Ablation resulted in (b) due to the multiple pulse exposure, despite a fluence below the single-pulse ablation threshold. The patterns recorded with this imaging technique are effectively identical to those produced through selective chemical etching followed by SEM imaging (as in Fig. 1).

the absorbed light. It may also be possible that the reaction force from the ablated material onto the surface of the crystal substrate produces a local electric field through the piezoelectric effect that assists the formation of domains at the elevated temperature. This domain nucleation is likely to initiate at structural defects and propagate along crystalline directions, forming the lines seen in Fig. 1.

To further elucidate the nature of these features, scanning force microscopy (SFM) was employed, operating in contact mode using a conductive probe tip to apply an ac voltage $\left(V_{p p}=10-20 \mathrm{~V}, f=35-38 \mathrm{kHz}\right)$. A cantilever with a large spring constant $\left(14 \mathrm{~N} \mathrm{~m}^{-1}\right)$ ensured limited interference from electrostatic effects. ${ }^{15}$ Hence, domain structures can be detected and readily characterized.

Imaging oppositely oriented domains is possible by SFM due to their $180^{\circ}$ out-of-phase responses. These responses can alternatively be viewed as in-phase, but of opposite sign (i.e., while one domain has a positive response, the opposite domain has a negative response). However, the rapid decay of the electric field away from the SFM tip means that the detected signal is dependent upon the size of the measured domains. Thus, for surface domains of approximately micron dimensions (as reported in Ref. 13) or larger, the amplitude response of the surface domain exceeds that of the bulk domain. The resulting detected signal is a summation of contributions from both domains, with phase response following that of the surface domain. In this case, contrast in both amplitude and phase is observed in the detected signal. Conversely, for smaller domains, including the size of the UVinduced features reported in this letter, the bulk amplitude contribution may exceed that from the surface domain, resulting in a detected signal with a phase response effectively following that of the bulk. Therefore, very little (if any) phase contrast is detected for these small features, but a recognizable reduction in the amplitude signal is still measured by the SFM.

The SFM scans of unetched $+z$ face of lithium niobate irradiated with 298 and $248 \mathrm{~nm}$ light [Figs. 3(a) and 3(b), respectively] show similar patterns to those revealed by selective chemical etching. Topography scans without an applied voltage confirmed that the lines of contrast in Fig. 3 do not correspond to topographical features prior to etching. Therefore, these UV-induced features display contrast in piezoelectric response relative to the surrounding areas and preferential etching compared to the surrounding unetched $+z$ face. Furthermore, the cross-sectional depth profile is very similar to that of surface domains formed through electric field over-poling. The evidence therefore leads to the conclusion that these line features correspond to near-surface domain-inverted material, induced by interaction of the incident UV light.

The domain depths of $\sim 2 \mu \mathrm{m}$ are compatible with waveguide dimensions, and the quality of the $-z$ domains as evidenced by etching appears high. The aim now is to induce such surface domain formation via phase-mask addressing geometries, or other interferometric techniques, to generate submicron periodic domain gratings. The measured domain widths of $150-300 \mathrm{~nm}$ are eminently compatible with structures with periods of twice this value, from 300 to $600 \mathrm{~nm}$, and this is exactly the range required for applications in UV quasi-phase-matching, electro-optic Bragg gratings, and photonic crystal structures. We foresee this technique as being of ready commercial applicability once sufficient degrees of control have been established on the final quality of the structures produced.

The authors are grateful to the Engineering and Physical Sciences Research Council (EPSRC) for research funding via Grant No. GR/S47373, and to INTAS for funding via Reference No. 03-51-6562. Financial support of the Deutsche Forschungsgemeinschaft is also gratefully acknowledged. Thanks also go to James Gates at the ORC, and to Lloyd Peto and Chengge Jiao from FEI, Bristol, UK, for FIB machining.

${ }^{1}$ Z. Zhiyong, Z. Yongyuan, Z. Shining, and M. Naiben, Phys. Status Solidi A 153, 275 (1996).

${ }^{2}$ S. Miyazawa, J. Appl. Phys. 50, 4599 (1979).

${ }^{3}$ M. Yamada and K. Kishima, Electron. Lett. 27, 828 (1991).

${ }^{4}$ M. Yamada, N. Nada, M. Saitoh, and K. Watanabe, Appl. Phys. Lett. 62, 435 (1993).

${ }^{5}$ V. Y. Shur, E. L. Rumyantsev, E. V. Nikolaeva, E. I. Shishkin, R. G. Batchko, G. D. Miller, M. M. Fejer, and R. L. Byer, Ferroelectrics 236, 129 (2000).

${ }^{6}$ G. Rosenman, P. Urenski, A. Agronin, Y. Rosenwaks, and M. Molotskii, Appl. Phys. Lett. 82, 103 (2003).

${ }^{7}$ V. Dierolf and C. Sandmann, Appl. Phys. Lett. 84, 3987 (2004).

${ }^{8}$ M. Müller, E. Soergel, and K. Buse, Appl. Phys. Lett. 83, 1824 (2003).

${ }^{9}$ M. Fujimura, T. Sohmura, and T. Suhara, Electron. Lett. 39, 719 (2003).

${ }^{10}$ C. L. Sones, C. E. Valdivia, J. G. Scott, S. Mailis, R. W. Eason, D. A. Scrymgeour, V. Gopalan, T. Jungk, and E. Soergel, Appl. Phys. B: Lasers Opt. (to be published).

${ }^{11}$ J. G. Scott, A. J. Boyland, S. Mailis, C. Grivas, O. Wagner, S. Lagoutte, and R. W. Eason, Appl. Surf. Sci. 230, 138 (2004).

${ }^{12}$ C. L. Sones, S. Mailis, W. S. Brocklesby, R. W. Eason, and J. R. Owen, J. Mater. Chem. 12, 295 (2002).

${ }^{13}$ A. C. Busacca, C. L. Sones, V. Apostolopoulos, R. W. Eason, and S. Mailis, Appl. Phys. Lett. 81, 4946 (2002).

${ }^{14}$ C. L. Sones, PhD thesis, University of Southampton, 2003.

${ }^{15}$ G. Hu, T. Tang, and J. Xu, Jpn. J. Appl. Phys., Part 1 41, 6793 (2002). 